\title{
Ruptured bronchial artery aneurysm mimicking aortic dissection
}

\author{
R. J. C. HALL, G. A. H. MILLER, AND I. H. KERR \\ From Brompton Hospital, Fulham Road, London
}

A patient is presented in whom rupture of a bronchial artery aneurysm produced a clinical picture indistinguishable from an aortic dissection. This case emphasises the importance of considering alternative diagnoses when a patient presents with symptoms typical of a particular condition.

We present a patient in whom the clinical picture closely mimicked aortic dissection, but in whom chest pain, a widened mediastinum, and left haemothorax resulted from haemorrhage from a bronchial artery aneurysm.

\section{Case report}

A 56-year-old man, who had had a chronic productive cough for many years, presented with sudden onset of severe tearing central chest pain radiating into his back. On examination he was sweating and in pain with a pulse of 110 and blood pressure of $140 / 80 \mathrm{mmHg}$. All peripheral pulses were normal and there was no evidence of aortic regurgitation. The electrocardiogram was normal and the chest $x$-ray film showed a greatly widened mediastinum, a small left pleural effusion and atelectasis, with consolidation at the left base (Fig. 1). During the next 24 hours the patient deteriorated with increasing hypotension and a repeat chest $x$-ray film showed complete opacification of the left hemithorax. Two litres of bright blood was aspirated from the left chest and the patient was transfused with blood, and then transferred to the Brompton Hospital with a preliminary diagnosis of aortic dissection.

\section{ANGIOGRAPHY}

The aorta was normal, with no evidence of dissection. A tortuous, greatly enlarged, bronchial vessel was observed running towards the left lower lobe and was selectively cannulated. There was an aneurysm on this vessel 2 to $3 \mathrm{~cm}$ from its origin (Fig. 2). Venous return from the area supplied by this vessel was into a pulmonary vein. There were several other smaller, but enlarged, bronchial arteries running to the left lower lobe. In view of the angiographic and clinical findings urgent operation was performed.

\section{OPERATIVE FINDINGS}

Left thoracotomy revealed $750 \mathrm{ml}$ fresh blood in the pleural cavity. There was a mass of tense extravasated blood contained in the fascia around the descending aorta and extending forward around the pericardium. The aorta was normal and the bleeding was coming from the bronchial arterial aneurysm shown at angiography. This was immediately below the point where the left vagus gives off its recurrent branch. There were other enlarged bronchial vessels running to the left lower lobe. A left lower lobectomy was performed from which he made an uneventful recovery.

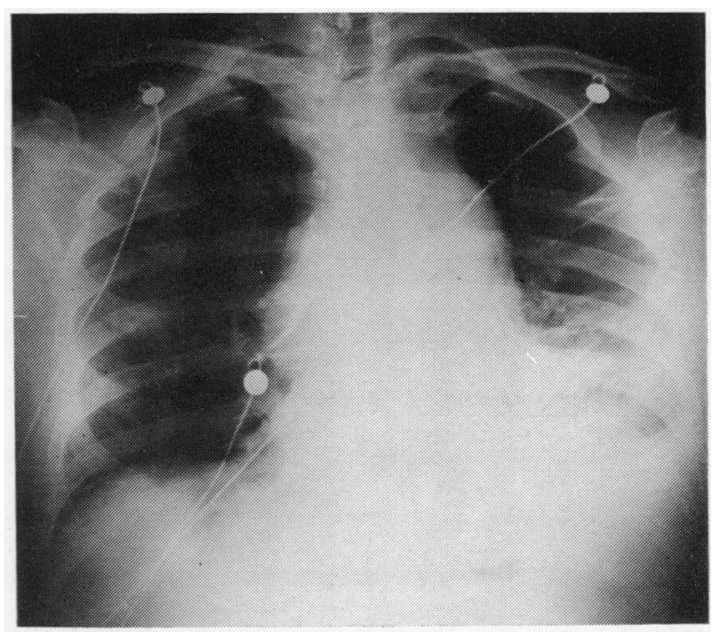

Fig. 1 Chest $\mathrm{x}$-ray film on admission to hospital. 
PATHOLOGICAL FINDINGS

Macroscopical examination showed severe bronchiectasis of the anterior and lateral lung segments of the left lower lobe, which were not sequestrated.

\section{Discussion}

Areas of bronchiectasis are often supplied by enlarged bronchial arteries (Turner-Warwick, 1963). Rupture of an aneurysm on such a vessel has not been reported previously though rupture of other intrathoracic aneurysms (De France et al., 1974) and pulmonary arteriovenous fistulae (Brummel-

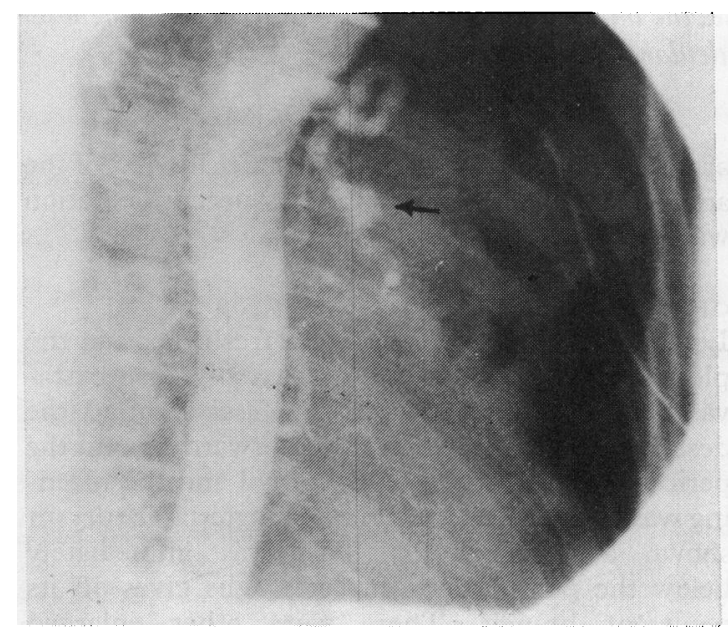

Fig. 2 Aortogram showing the large bronchial artery and the aneurysm on it (marked by an arrow). kamp, 1961; Dalton et al., 1967) may present with haemothorax.

This patient presented with a clinical picture frequently seen with aortic dissection though the chest pain and mediastinal widening resulted from blood stripping around the aorta from an 'extraaortic' vessel. This case emphasises the importance of considering alternative diagnostic possibilities, some of which are amenable to urgent surgery, when faced with an apparently typical clinical picture of dissection. It also emphasises that early investigation of suspected aortic dissection is mandatory, both to aid correct management of dissection and to exclude other conditions which may need urgent surgery.

We would like to thank Mr. M. Paneth, who operated on this patient, for his permission to report this case.

\section{References}

Brummelkamp, W. H. (1961). Unusual complication of pulmonary arteriovenous aneurysm: intrapleural rupture. Diseases of the Chest, 39, 218-221.

Dalton, M. L., Goodwin, F. C., Bronwell, A. W., and Rutledge, R. (1967). Intrapleural rupture of pulmonary arteriovenous aneurysm. Diseases of the Chest, 52, 97-100.

De France, J. H., Blewett, J. H., Ricci, J. A., and Patterson, L. T. (1974). Massive hemothorax: two unusual cases. Chest, 66, 82-84.

Turner-Warwick, M. (1963). Systemic arterial patterns in the lung and clubbing of the fingers. Thorax, 18, 238-250.

Requests for reprints to Dr. G. A. H. Miller, Brompton Hospital, Fulham Road, London SW3 $6 \mathrm{HP}$. 